\title{
Medical cannabis: strengthening evidence in the face of hype and public pressure
}

\author{
Mary-Ann Fitzcharles MBChB, Yoram Shir MD, Winfried Häuser MD
}

Cite as: CMAJ 2019 August 19;191:E907-8. doi: 10.1503/cmaj.190509

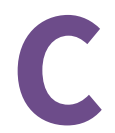

anada has been at the forefront of cannabis research, education and regulation for the past 2 decades, yet uncertainty remains about how the drug should be used in medicine. Physicians lack evidence-based information and formalized training about cannabis, which stems, in part, from the drug's previously illegal status that hindered research. Among the public, however, many perceive cannabis as a natural and safe medical treatment. Patients increasingly seek advice about cannabis from physicians, request prescriptions or experiment with cannabis for medical problems on their own. However, physicians must adhere to good medical practice regardless of public pressure and provide counselling to patients based on up-to-date knowledge and evidence. Now that cannabis is legal in Canada more research should be forthcoming, but the evidence base remains weak.

Medical cannabis is unique in that it bypassed the process of due diligence required for drug registration and entered the therapeutic domain buoyed mostly by advocacy. ${ }^{1}$ Positive effects of cannabinoids have been reported for severe childhood epilepsy and chemotherapy-induced nausea, and as palliation at end of life, but data are limited. ${ }^{2}$ For other conditions commonly believed to improve with cannabis use, such as pain relief or mood disorder, the evidence is less convincing. ${ }^{1,3,4}$ A 2018 systematic review and meta-analysis of the effect of cannabis-based medicines on chronic noncancer pain reported a number needed to benefit of 24 , whereas the number needed to harm was $6 .{ }^{3} \mathrm{~A}$ randomized controlled trial (RCT) in the Netherlands that involved women with fibromyalgia who were treated with pharmaceutical-grade inhaled cannabis in various concentrations of $\Delta 9$-tetrahydrocannabinol (THC) and cannabidiol (CBD) found that none of the treatments had an effect greater than placebo on spontaneous pain, and reduced pain scores were significantly correlated with the extent of drug high. ${ }^{5}$ Furthermore, CBD increased plasma concentrations of THC but reduced THC's analgesic effects, which emphasizes the complexity of THC-CBD interactions and the possible role of psychotropic mechanisms on symptom relief.

Patients often seek treatments that offer better symptom relief than their current medications. Some hope that medical cannabis could be a less-harmful alternative to opioid medication and could aid harm reduction. The perception of cannabis as being safer than opioids may have been reinforced by its recent legalization for recreational use in some jurisdictions. Media pro-

\section{KEY POINTS}

- A lack of evidence and formalized training means that physicians remain uncertain as to how cannabis can be used to treat medical conditions.

- The legalization of recreational cannabis and overwhelming marketing may have primed some patients to believe in its effectiveness for many indications.

- Self-styled medical and nonmedical experts project an image of knowledge and a promise of personalized medicine using one product that runs counter to our understanding of competent medical care.

- Governments and regulators worldwide have a moral obligation to support the scientific study of cannabis to protect the wellbeing of patients.

motion, replete with images of pristine cultivation facilities, personal testimony and vocal medical advocacy, has done much to influence this perception. Patients are often primed by the media to believe medical cannabis is a valid treatment for many symptoms. Medication beliefs influence treatment adherence and efficacy, with personal choice reinforcing positive expectations. ${ }^{6}$ The ability to choose a treatment and manipulate dosing or method of administration may foster a sizable placebo effect, which may be further reinforced by financial commitment when there is no reimbursement. It is possible that medical cannabis does not meaningfully affect the medical condition other than through subtle psychoactive effects that bring comfort. ${ }^{5}$

Patients who want to try cannabis as a treatment often seek medical advice about dosages, choice of specific products and method of administration. ${ }^{7}$ Other than broad recommendations that cannabis should not be smoked and to use products with low THC and high CBD levels, no regulatory or medical body has provided specific guidance. ${ }^{4}$ This provides the perfect setting for an emergent medical cannabis industry, with self-styled medical and nonmedical experts projecting an image of knowledge and promise of personalized medicine. Although more than $90 \%$ of dispensary staff in the United States reported providing advice to patients, only $20 \%$ had (unspecified) medical training and $13 \%$ had scientific training. ${ }^{8}$ The medical advice that was given was often not evidence based. 
Many people feel secure in a medical setting, which has opened the door for cannabis clinics staffed by physicians who are savvy about cannabis. Yet the concept of a physician focusing treatment strategies on a single product is peculiar and contrary to medical care standards. The notion that a "cannabis expert" can identify the ideal strain or molecular content for a specific condition for a specific patient is not backed by concrete evidence. Furthermore, the accuracy of the labelling of cannabis products is questionable, and regulations regarding both quality control and labelling are insufficient. ${ }^{9}$ To save costs, many patients may choose to access cannabis illegally, compounding risks related to unknown molecular content, quality and safety, especially since the concentration of THC offered as a medicinal product by Canadian growers has reportedly increased in recent years. ${ }^{10}$

Despite advocacy, politics and commerce having outpaced sound clinical evidence regarding the use of medical cannabis, physicians must provide advice based on valid science, not pseudoscience or hype. Therefore, there is an urgent need to generate better evidence about the benefits and risks of medical cannabis. Preclinical study should be used as a guide to identify molecular compositions of cannabis that may be effective for a specific indication and warrant further testing in the clinical setting. The gold standard of RCTs may, however, be less realistic for accumulating evidence for using medical cannabis for reasons that include variability in molecular content (even within specific strains of the plant product), current prevalent use by many patients, issues of effective blinding and the need for longterm study.

Observational studies involving cohorts of patients being managed in usual clinical care, but not those conducted by cannabis growers or in cannabis clinics or dispensaries, will generate real-world information. Investigator-initiated studies that are financially supported by the cannabis industry should adhere to the regulatory standards required for pharmaceutical products.

Governments and regulators worldwide have a moral obligation to support the scientific study of cannabis to protect the well-being of patients and avert a potential disaster similar to the opioid epidemic in North America. Although some patients may benefit from cannabis in the short term, long-term consequences of its use are not yet known. ${ }^{11}$ Until the evidence base is strong enough to support sound decisions about the use of cannabis as a medical treatment, the well-being of patients will continue to come second to profit-motivated parties.

\section{References}

1. D'Souza DC, Ranganathan M. Medical marijuana: Is the cart before the horse? JAMA 2015;313:2431-2.

2. The health effects of cannabis and cannabinoids: the current state of evidence and recommendations for research. 2017, Washington (D.C.): The National Academies Press; 2012.

3. Stockings E, Campbell G, Hall WD, et al. Cannabis and cannabinoids for the treatment of people with chronic noncancer pain conditions: a systematic review and meta-analysis of controlled and observational studies. Pain 2018; 159:1932-54.

4. Allan GM, Ramji J, Perry D, et al. Simplified guideline for prescribing medical cannabinoids in primary care. Can Fam Physician 2018;64:111-20.

5. van de Donk T, Niesters M, Kowal MA, et al. An experimental randomized study on the analgesic effects of pharmaceutical-grade cannabis in chronic pain patients with fibromyalgia. Pain 2019;160:860-9.

6. De Las Cuevas C, de Leon J. Reviving research on medication attitudes for improving pharmacotherapy: focusing on adherence. Psychother Psychosom 2017;86:73-9.

7. Fitzcharles MA, Niaki OZ, Häuser W, et al. Position statement: a pragmatic approach for medical cannabis and patients with rheumatic diseases. J Rheumatol 2019;46:532-8.

8. Haug N, Kieschnick D, Sottile JE, et al. Training and practices of cannabis dispensery staff. Cannabis Cannabinoid Res 2016;1:244-51.

9. Bonn-Miller MO, Loflin MJE, Thomas BF, et al. Labeling accuracy of cannabidiol extracts sold online. JAMA 2017;318:1708-9.

10. Mammen G, de Freitas L, Rehm J, et al. Cannabinoid concentrations in Canada's regulated medical cannabis industry. Addiction 2017;112:730-2.

11. Häuser W, Finnerup NB, Moore RA. Systematic reviews with meta-analysis on cannabis-based medicines for chronic pain: a methodological and political minefield. Pain 2018;159:1906-7.

Competing interests: Winfried Häuser has received reimbursement of travel fees and accomodations by Bioevents for co-organizing congresses (Controversies on Cannabis-Based Medicines) outside the submitted work. No other competing interests were declared.

This article has been peer reviewed.

Affiliations: Division of Rheumatology (Fitzcharles), McGill University Health Centre; Alan Edwards Pain Management Unit (Fitzcharles, Shir), McGill University Health Centre, Montréal, Que.; Health Care Center for Pain Medicine and Mental Health (Häuser), Saarbrücken St. Johann, Saarbrücken, Germany; Department of Psychosomatic Medicine and Psychotherapy (Häuser), Technische Universität München, München, Germany

Contributors: All of the authors contributed substantially to the conception and design of the work, participated in the writing of the manuscript, reviewed it critically for important intellectual content and agreed to be accountable for all aspects of the work.

Correspondence to: Mary-Ann Fitzcharles, mary-ann.fitzcharles@ muhc.mcgill.ca 